\title{
A. QUANTITATIVE MEASURE OF MANDIBULAR JOINT DYSFUNCTION: PHASE PLANE MODELLING OF JAW MOVEMENT IN MAN
}

\author{
W. D. McCall JR., J. O. Balley JR. and M. M. Ash JR. \\ Biocngineering Laboratory, Department of Occlusion and Dental Research Institute, \\ School of Dentistry, University of Michigan, Ann Arbor, Michigan 48109, U.S.A.
}

\begin{abstract}
Summary-Jaw movement during the open-close-clench cycle was recorded using a Hall-effect generator and a small permanent magnet affixed to opposing incisor teeth. Computer programs provided graphic display of jaw-closing velocity as a function of jaw position (the phase plane) and determined the error between the experimental data and a mathematical model. A large error indicated dysfunction and predicted successful treatment by accepted occlusal therapy; a small error indicated normal function or predicted unsuccessful occlusal treatment. These otservations suggest that phase plane modelling provides a quantitative measure of joint dysiunction that may be used in monitoring treatment progress and in separating clinically diagnosed dysfunction into two categories - one which responds to occlusal therapy, and one which does not.
\end{abstract}

\section{INTRODUCTION}

Several controversies are associated with temporomandibular joint (TMJ) and masticatory muscle dysfunction. One controversy involves nomenclature where various names are attached to what is presumed to be a single clinical entity. These names include TMJ pain dysfunction syndrome (Schwartz, 1956), myofascial pain dysfunction syndrome (Greene and Laskin, 1971), mandibular pain dysfunction syndrome (Molin, 1973), and traumatic TMJ arthritis (Ramfjord and Ash, 1971). More serious controversies involve the aetiological theories and resultant treatment. It has been proposed that the aetiology lies solely with masticatory muscles (Schwartz and Tausig, 1954), occlusion (Dawson, 1974), joint (Weinberg, 1972), referred pain (Travell, 1960), psyche (Kydd, 1959; Luoton, 1969), or with combinations of these factors (Ramfjord and Ash, 1971). The subsequent treatment modalities then involve such diverse treatments as exercise (Carlsson et al., 1970), intramuscular injection of anaesthetic (Schwartz and Tausig, 1954), drugs (Greene and Laskin, 1971), alterations of the osclusion (Ramfjord and Ash, 1971; Dawson, 1974) and psychological counselling (Moulton, 1966; Pomp, 1974). These controversies have recently been critically reviewed (DeBoever, 1973). The prevalence of the cardinal symptoms in a clinical population (Carraro, Caffesse and Albano, 1969) indicates general agreement on the clinical symptoms, while population studies (Agerberg and Carlsson, 1972; W. K. Solberg, M. W. Woo and J. B. Houston, Personal communication) indicate that about 8 per cent of the general population report pain. At least one symptom exists in 60 to 70 per cent of the population. The prevalence and types of occlusal interferences in a symptomatic clinical population are also available (Geering, 1974).

A clinical index based on quantitative diagnostic parameters would be valuable in assessing symptom severity, treatment progress and comparing different treatments, such an approach might provide insight into the underlying aetiological mechanisms. Our present purpose is to report one such diagnostic parameter based on fluctuation of jaw-closing velocity.

\section{Transducer}

\section{METHODS}

Jaw motion was registered by cementing a small permanent magnet on a mandibular incisor and recording its magnetic field at a maxillary incisor with a magnetic field-sensing device (Kydd, Harrold and Smith, 1967; Bando et al., 1972; Woltjen et al., 1973; Jankelson et al., 1975). The device was a Hall-effect generator (Model HI-5, American Aerospace Controls, Farmington, N.Y.). It was sensitive to the static magnetic field and was not influenced by saliva, tongue, food bolus etc, in the intervening space. The output of the generator was amplified and stored on analogue magnetic tape as part of a larger series of experiments. Typical raw data, including concurrent electromyographic (EMG) traces which will not be discussed here, are given in Fig. 1.

\section{Data processing}

The analogue tape was replayed at one-quarter speed and the jaw-motion signal was sampled at a rate of 1000 samples per sec for $2 \mathrm{sec}$ by analogue-todigital (A/D) converter. The digital representation of each sample was stored in the memory of a laboratory minicomputer (Geister et al., 1975) (Fig. 2.)

A computer program, written in Basic language, performed the following functions: (1) Recorded and incorporated calibration points. (2) Sampled the signal. (3) Previewed or presented the signal on the X-Y plotter for verification. (4) As the magnetic field decayed non-linearly with increasing distance, part of the computer program was used to linearize and calibrate the data. (5) Smoothed the position signal to reduce noise. (6) Digitally computed velocity of jaw closing. (7) Smoothed the velocity signal. (8) Plotted 

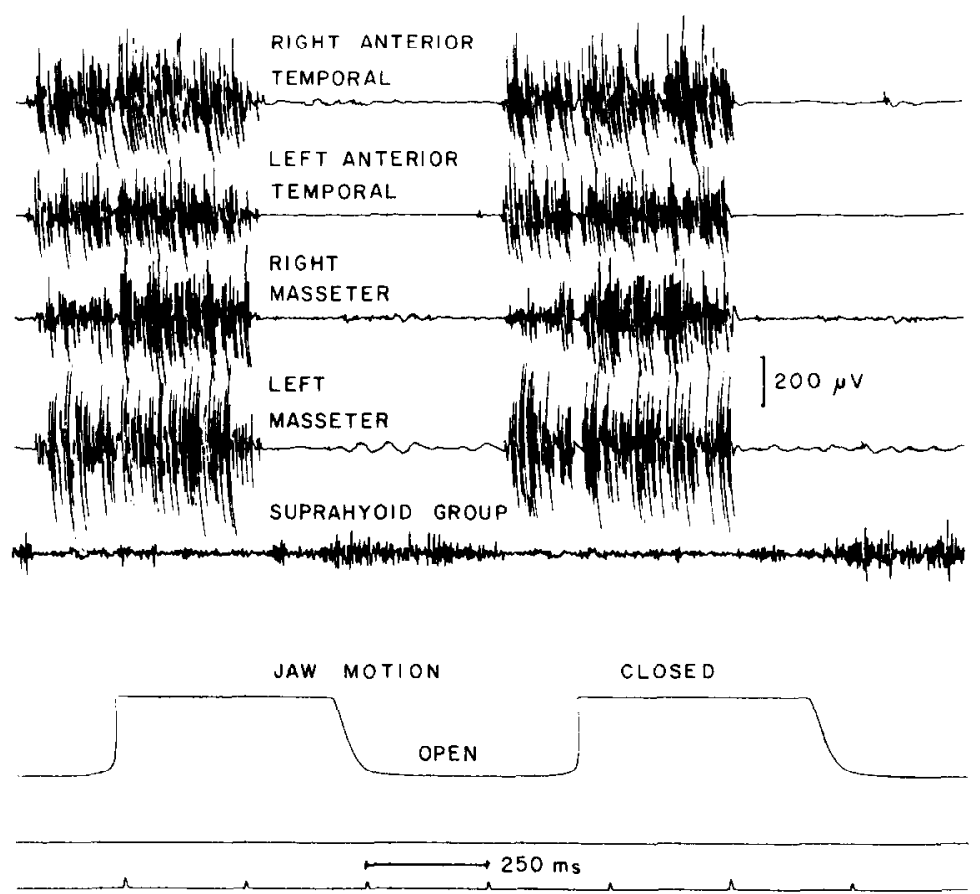

Fig. 1. Typical raw data. Five electromyographic channels and the output of the amplifier associated with the Hall-effect generator from a normal subject. As the position output is non-linear with respect to distance, measurements cannot be made directly from the tracing. The opening and closing can be observed, however.

the velocity as a function of position, called the "phase plane" in engineering parlance. This term is explained below. (9) Computed the coefficients of a parabola to best fit the experimental data. (10) Plotted the parabola using the coefficients generated above. (11) Computed the root-mean-square (rms) error between the experimental data and the parabolic model. This computation was normalized by the number of data points used in the plot and by the maximum velocity predicted by the parabola. Thus the rms error was presented as a percentage of the maximum velocity predicted by the parabola. This percentage error was used as the diagnostic parameter. Machine language subroutines were written for input from the A/D converter and output to the $\mathrm{X}$-Y plotter.

The term "phase plane" arose in the study of nonlinear differential equations and their engineering applications (Hsu and Meyer, 1968). There, the term denotes a plot of any dependent variable vs. its time derivative. Here, phase plane refers specifically to the plot of jaw position vs. jaw velocity. It seemed to us better to use a term from another discipline rather than generate new jargon.

The method of computing the error between the experimental data and parabolic model was open to choice. As both positive and negative errors can occur, some method that ignores the sign must be used. The average absolute error, for example, might be used. However, since the best fit of the parabola to the data was obtained by the method of least-squareerror, it seemed natural to express the error in a similar way. These considerations led to the choice of root-mean-square (rms) error.

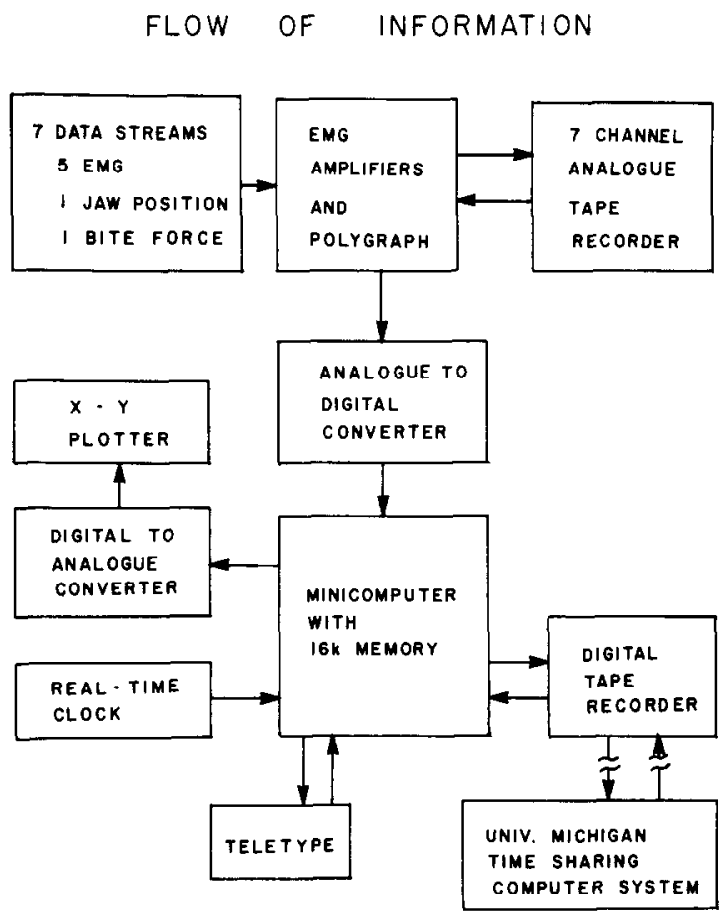

Fig. 2. Block diagram of computer system. The information flows into the computer via the analogue-to-digital (A/D) converter (Datel Model 256). The data are processed by the minicomputer (Computer Automation Inc., Model Alpha 16). The plots are generated on an X-Y plotter (Moseley Model 2DR-2M) after emerging from the computer via the $D / A$ converter. Textual data are presented on the teletype. 
Each of the steps in the computer program were made interactive to permit monitoring of the procedure. For example, the data were previewed to see if the closing movement was successfully sampled; if not, it was resampled. All data were not relevant because many data points may have been prior to or after the closing stroke. These were not used in the computations. The scale factors used in plotting the phase plane were entered manually. Teletype control enabled clinical personnel untrained in the intricacies of computers to utilize the program efficiently.

\section{Material}

Subjects were selected from the staff, students and patients of the University of Michigan School of Dentistry. An initial examination determined the presence or absence of the signs and symptoms of masticatory dysfunction: join: sounds (clicking and crepitation), limited jaw opening, muscle or TMJ tenderness and pain in the TMJ area or muscles of mastication. Subjects were categorized as normal if they presented no signs or symptoms and reported no history of disturbances of the masticatory system.

The data from 9 clinically normal subjects and from 13 patients clinically diagnosed as having masticatory dysfunction were analyzed. Of the 13,9 were responsive to treatment. The other 4 patients were refractory to treatment.

\section{Clinical procedure's}

The subject was seated in a dental chair with head support and with the ala-tragus plane horizontal. The Hall-effect generator and magnet were cemented in place. Because distance and alignment differed from one mounting to the next, a series of calibration points was recorded. As part of the larger investigation, muscular activity was also monitored using surface electrodes on the suprahyoid group and bilaterally on the masseters and anterior temporal muscles. During each recording session, the subject was asked to perform the open-close-clench cycle 10 times (Griffin and Munro, 1969).

For the subjects classified as dysfunctional, this procedure was performed prior to delivery of an occlusal splint (Ramfjord and Ash, 1971), during the splint adjustment period and upon cessation of the clinical symptoms. For normal subjects the procedure was repeated at similar time intervals.

\section{RESULTS}

A typical phase-plane trajectory (a plot of jaw closing velocity vs. jaw position) from a normal subject started at an open jaw position and zero velocity (Fig. 3). As the distance decreased toward closing, the magnitude of the velocity increased smoothly to a maximum, and then rapidly decreased to zero at tooth contact. The rms error in normal subjects ranged from 10.5 to 18.7 per cent.

A typical phase plane trajectory from a dysfunctional patient prior to treatment by an occlusal splint showed large alterations of the velocity during closing (Fig. 4A).

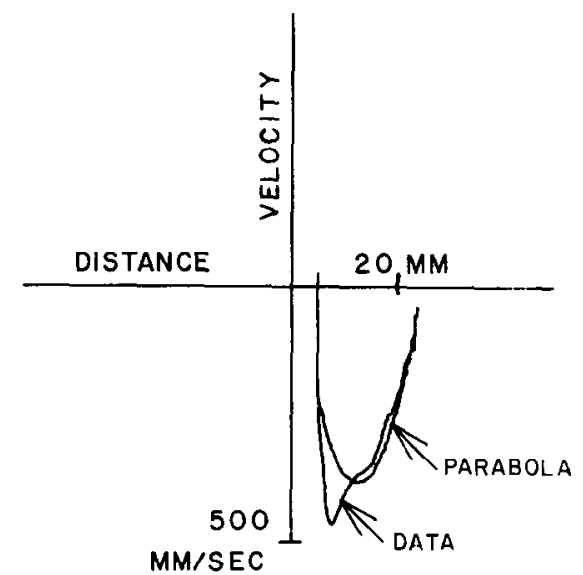

Fig. 3. Phase plane trajectory from a normal subject. Both the experimental data and the parabolic model are shown. The repeatability was such that successive trajectories were usually within one per cent error of each other. The rms error was 10.5 per cent.

After successful splint treatment, the amplitude of the velocity excursions was markedly attenuated compared to the pre-treatment trajectories (Fig. 4B). The error ranged from 7 to 17.5 per cent for successfully treated patients. The criterion for successful treatment was cessation of symptoms for a period of at least one month; these patients were then considered to be clinically normal.

Histogram of the rms error between the parabolic model and the experimental data (Fig. 5) showed that normal subjects and successfully treated patients fell, without exception, in the range of less than 20 per cent. The dysfunctional patients who were subsequently treated successfully by an occlusal splint showed, with one exception, a phase plane error at the pretreatment recording session which exceeded 20 per cent.

There were four exceptions to the general finding that patients with symptoms exceeded 20 per cent error and asymptomatic patients showed less than 20 per cent error. These patients were clinically diagnosed as having masticatory dysfunction but showed rms errors that would place them in the normal range of the histogram. The dysfunctional patients with large rms errors showed cessation of symptoms within a few weeks or even days with splint therapy; however, the four with small initial rms errors were refractory to the splint treatment for several months. Thus, by the method we report, it is possible to separate the dysfunctional patients into two groups: those who can be successfully treated and those who cannot be successfully treated by occlusal splint therapy alone.

The data were examined statistically by the Fisher Exact Probability Test (Blalock, 1960). Using the data depicted in Fig. 5 , a 2 by 2 contingency table was constructed according to whether the phase plane error was greater than or less than 20 per cent. The asymptomatic patients (normal plus post-treatment) vs. symptomatic patients (pretreatment plus refractory) differed with statistical significance at the 0.001 level. 

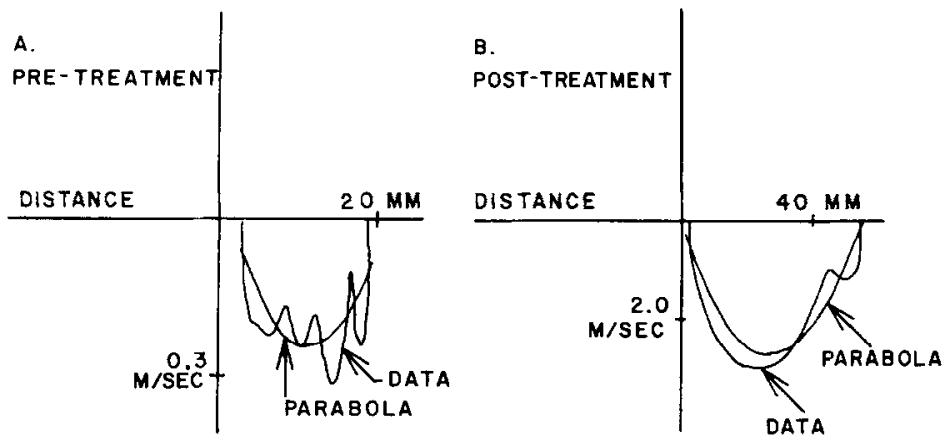

Fig. 4. Phase plane trajectories from dysfunctional patient. A. Prior to treatment. The rms error was 24 per cent. B. Subsequent to treatment. The rms error was 15.4 per cent.

To compare the rms error between the normal subjects and the dysfunctional patients who were subsequently successfully treated, a Mann-Whitney $U$ test (Siegel, 1956) was performed. It showed that the two groups differed with statistical significance $(p<0.001)$. Further, the data from the dysfunctional patients where both pre-treatment and post-treatment data existed were subjected to the Wilcoxon matchedpairs signed-ranks test (Siegel, 1956) (Fig. 5 inset). This test showed a statistically significant difference $(p<0.01)$.

\section{DISCUSSION}

\section{Technical}

The parabola as the model to be fitted to rather than a higher order polynomial or some other form of model was chosen mainly for simplicity and to

SUMMARY OF PHASE PLANE RESULTS

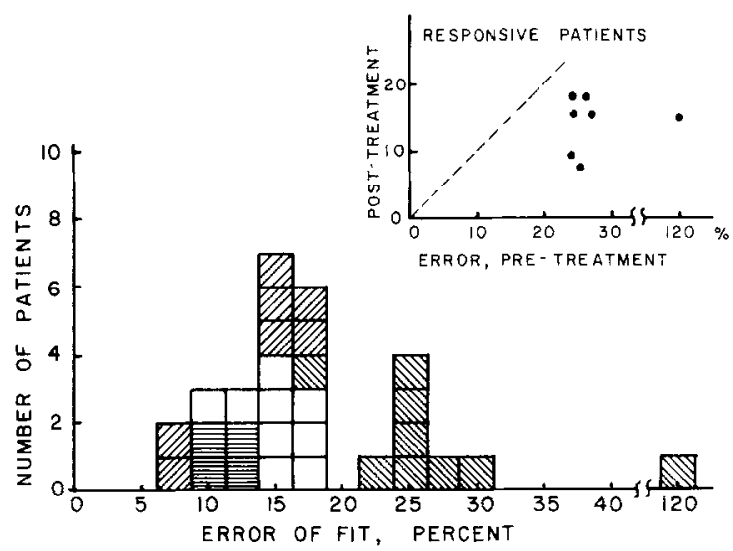

Fig. 5. Histogram of phase plane results showing the number of patients at each discrete value of error between the experimental data and the parabolic model. Data from clinically normal subjects are given as open squares. Hatching sloping down to the right indicates the pre-treatment dysfunctional patients who were subsequently treated successfully by occlusal techniques. Hatching sloping down to the left indicates these same patients following successful treatment and cessation of symptoms. Horizontal hatching indicates patients who exhibited clinical symptoms but were refractory to occlusal splint treatment. Inset: Pre- and post-treatment data from patients responsive to occlusal therapy. If no change occurred in the phase plane error, all data points would fall on the dashed reference line. meet the need for a model that would separate the normal subjects from the dysfunctional patients. The model has served well.

The characteristics of jaw closure in the present study require some understanding of the processing techniques utilized. The low pass filtering of the smoothing routines distorted the trajectory to some extent and so reduced the oscillations observed in the dysfunctional patients.

\section{Clinical}

It should be stressed that the idea of an "ideal" patient fitting an "ideal" parabola was not intended; the parabola was used only for convenience. There is no rational basis for attaching any clinical significance to the parabolic shape.

The mechanism which caused the velocity alterations in the dysfunctional patients is of great interest as it might provide some insight into the aetiology of the individual problem. The possible mechanisms include mechanical impediment in the joint or its capsular structures, alteration of muscular activity and peculiarity in the transducer system. Each may be excluded by the appropriate experiments: The contention that the velocity alterations arose wholly within the transducer system can be rejected because the device was monotonic in static calibration and had no dynamic properties to cause such a phenomenon. Moreover, normal subjects exhibited smooth velocity increases and dysfunctional patients who had been successfully treated exhibited markedly attenuated velocity alterations.

However, because of the roughly spherical shape of the magnetic field, lateral movements were inter preted as low or zero velocity at a constant position. Thus, the possibility exists that the velocity alter ations' were in fact movements with a large lateral and small vertical component. $H$. Graf (personal communication) has data from one dysfunctional patient showing lateral movement during a snap close. Jankelson et al. (1975), however, illustrate an instance in which similar velocity alterations did occur in a purely vertical plane. To investigate such a possibility, a second dimension of jaw motion must be registered. Design of a two-dimensional system is proceeding. We emphasize, however, that, whatever the outcome, the clinical significance of our present findings remains unchanged. 
Alterations in r.1uscle activity would be observed in the simultaneous electromyographic tracings if techniques were de:veloped to correlate the EMG with the jaw motion traces. We have not done so yet.

Regarding impediment in the joint itself, the possibility exists that the mechanisms causing clicking and crepitation are responsible for the velocity alterations on a purely mechanical basis.

Given previous separation by clinical examination into symptomatic and asymptomatic groups, the jaw motion technique we report here may provide a further separation of the symptomatic patients into two groups: Those who can and those who cannot be successfully treated by an occlusal splint. The phase plane method may also provide a quantitative method for monitoring the treatment progress of those dysfunctional patients who are responsive to occlusal techniques. It provides objective evidence, to support the subjestive report of the patient, that the treatment has been successful.

Our results suggest the possibility that what is generally thought to be one disease entity might in fact be several.

The technique does not, however, distinguish between the two groups of asymptomatic patients: those who do and those who do not report a history of dysfunction $(\mathrm{U}=32.5, p>0.05)$. This confirms the clinical assertion that successful treatment makes these two groups indistinguishable.

Acknowledgement--This work was supported by USPHS Grant DE 02731.

\section{REFERENCES}

Agerberg G. and Cerrlsson G. E. 1972. Functional disorders of the masticatory system I. Distribution of symptoms according to age and sex as judged from investigation by questionaire. Acta odont. scand. 30, 597-613.

Bando E., Fukushirna S., Kawabata H. and Kohno S. 1972. Continuous observations of mandibular positions by telemetry. J. prosth. Dent. 28, 485-490.

Blalock H. M. Jr. 1960. Social Statistics, McGraw-Hill, New York

Carlsson G. E., Helkimo E., Helkimo M., Hellsing G., Jutler P. O. an d Osterberg T. 1970. Käkledsbesvar. Svensk Tandläk. Tidskr. 62, 1113-1118.

Carraro J. J., Caffesse R. G. and Albano E. A. 1969. Temporomandibular joint syndrome, a clinical evaluation. Oral Surg. 28, 54-62.

Dawson P. E. 1974, Evaluation, Diagnosis, and Treatment of Occlusal Prob'ems. Chap. 3. Mosby, St. Louis.
DeBoever J. A. 1973. Functional disturbances of the temporomandibular joint. Oral Sci. Rev. 2, 100-117.

Geering A. H. 1974. Occlusal interferences and functional disturbances of the masticatory system. J. Clin. Periodont. 1, 112-119.

Geister D. E.. McCall Jr. W. D. and Ash Jr. M. M. 1975. Computerized data acquisition and analysis for real time electromyography in clinical dentistry. Proc. Instn. elect. electron. Engrs. 63, $1404-1414$.

Greene C. S. and Laskin D. M. 1971. Meprobamate therapy for the myofascial pain-dysfunction (MPD) syndrome: a double-blind evaluation. J. Am. dent. Ass. 82, $581-590$.

Griffin C. J. and Munro R. R. 1969. Electromyography of the jaw-closing muscle in the open-close-clench cycle in man. Archs oral Biol. 14. 141-149.

Hsu J. C. and Meyer A. U. 1968. Modern Control Principles and Applications. Chap. 4. McGraw-Hill, New York.

Jankelson B., Swain C. W., Crane P. F. and Radke J. C. 1975. Kinesiographic instrumentation: a new technology. $J$. Am. dent. Ass. 90, 834-839.

Kydd W. L., 1959. Psychosomatic aspects of temporomandibular joint dysfunction. $J$. Am. dent. Ass. 59, 31-44.

Kydd W. L., Harrold W. and Smith D. E. 1967. A technique for continously monitoring the interocclusal distance. $J$. prosth. Dent. 18, 308-310.

Lupton D. E. 1969. Psychological aspects of TMJ dysfunction. J. Am. dent. Ass. 79, 131-136.

Molin C. 1973. Studies in mandibular pain dysfunction syndrome. Swed. Dent. J. 66, (Suppl. 4), 1-55.

Moulton R. E. 1966. Emotional factors in non-organic temporomandibular joint pain. Dent. Clin. N. Am. 609-620.

Pomp A. M. 1974. Psychotherapy for the myofascial paindysfunction (MPD) syndrome: a study of factors coinciding with symptom remission. J. Am. dent. Ass. 89, 629-632.

Ramfjord S. P. and Ash Jr. M. M. 1971. Occlusion. 2nd ed., Saunders, Philadelphia.

Schwartz L. L. 1956. A temporomandibular joint pain-dysfunction syndrome. J. chron. Dis. 3, 284-293.

Schwartz L. L. and Tausig D. P. 1954. Temporomandibular joint pain-treatment with intramuscular infiltration of tetracaine hydrochloride: a preliminary report. $N$. $Y$. St. dent. J. 20. 219-223.

Siegel S. 1956. Nonparametric Statistics for the Behavioral Sciences. McGraw-Hill, New York.

Travell J. 1960. Temporomandibular joint pain referred from muscles of the head and neck. J. Prosth. Dent. 10, $745-763$.

Weinberg L. A. 1972. Correlation of temporomandibular dysfunction with radiographic findings. J. Prosth. Dent. 28, 519-539.

Woltjen J. A., Timm G. W., Waltz F. W. and Bradley W. E. 1973. Bladder motility detection using the Hall effect IEEE Trans. Biomed. Engin. BME-20, 295-299. 